\title{
Face recognition with positive and negative samples using support vector machine
}

\author{
A.D.Chitra ${ }^{1 *}$ and P.Ponmuthuramalingam ${ }^{2}$ \\ Assistant Professor, Department of Computing, Coimbatore Institute of Technology, Coimbatore, India ${ }^{1}$ \\ Associate Professor and Head, PG \& Research Department of Computer Science, Government Arts College \\ (Autonomous), Coimbatore, India ${ }^{2}$
}

\section{(02016 ACCENTS}

\begin{abstract}
One of the major problems in the face classification comes from a large image variance caused by unknown pose of the recognized face. A huge amount of real-world applications for face detection exist. Modern-day work even proposes that any specific detectors can be approached by means of fast detection classifiers. In this research work, support vector machine (SVM) algorithm detects face from the input image with less amount of false detection rate. This algorithm uses the concept of recognizing edges, color skin and extracting features from the face. The result is maintained by the parameters describing the parts of the face. The research implements the highly powerful concept of SVM that is used for the classification of images. This classification is based on the training data set and test data set with positive and negative samples.

\section{Keywords}

Face detection, SVM algorithms, Edges detection, Classification, Positive and negative samples.
\end{abstract}

\section{Introduction}

In computer science, it also covers many different sub-areas such as face detection, face tracking, feature extraction, etc. These feature values depended on the detection of geometric facial features, including the distance and angles between points such as eye corners, mouth extremities, nostrils and chin top. Digital image processing is concerned with the development of computer algorithms working on digitized images [1]. Digital Image Processing concerns the following field of sciences viz pattern recognition, optics, signal processing, electronics, cognitive science and perception science. The goal of image processing is usually automatic detection or recognition of image content, in which case one can speak of machine vision [2]. This pipeline consists of the steps of pre-processing, feature extraction, segmentation, object recognition and image understanding [3]. In each step, the input and output data could either be images (pixels), measurements in images (features), and decisions made in previous stages of the chain (labels) or even object relation information (graphs) [4].

\footnotetext{
*Author for correspondence
}

In this paper we tested face recognition using SVM algorithm with trained and test database samples.

\section{Literature review}

A method of automatically generating one or more labels to describe the content of an image. Images are usually identified with the labels that are extracted from their low level features [5]. Machine learning techniques such SVM, Bayesian Networks, Artificial Neural Networks, Decision Tree and Composite Classifiers such bagging and boosting have facilitated image annotation by learning the correlations between image features and annotated labels.

Distributed SVM-A SVM kernel usually involves an algorithmic complexity of $O(m 2 n)$, where $n$ is the dimension of the input and $m$ represents the training instances [6, 7]. The computation time in SVM training is quadratic in terms of the number of training instances. In order to improve the efficiency large data is divided into small chunks and later integrated using a cluster of computers [8]. The training of SVM is computationally is intensive when the data size is large, this turns out to be a drawback.

Distributed Multiclass SVM-Due to various complexities, a direct solution to multiclass problems using a single step SVM training is usually avoided A 
superior approach is to combine a number of binary SVM classifiers to solve a multiclass problem $[9,10]$. Various approaches have been proposed such as one against rest (OAR), one against one (OAO) and decision trees based multiclass SVM techniques.

SVM Ensemble-The combination of classifiers leads to significant reduction of classification error in a wide range of applications. Although there are lot of classifiers, SVM ensemble shows more accuracy yet at the cost of intensive computation when the number replicated sample is large. Ensemble learning is extremely computational intensive which limits their applications in real environments $[11,12]$.

Distributed Multiclass SVM-This is based on single optimization technique. The notable advantage of this technique is that the training is a single step process thereby reducing the processing time. Keerthi et al is a dual method based on direct multiclass formulations of linear SVM [13]. Here the dual variables are optimized while parsing the dataset sequentially. This becomes difficult for a large optimization problem so we create a multiclass classifier based on the combination of binary classifiers [6]. The OAR method is one of the popular methods to solve multiclass problems in which a binary classifier is trained for each class, which separates a single class from the rest of the classes and then combines the classifiers for multiclass inference [14]. OVR can achieve high accuracy in classification but the training process is not efficient due the involvement of all training data for creating binary classifiers for each class.

\section{SVM Algorithm}

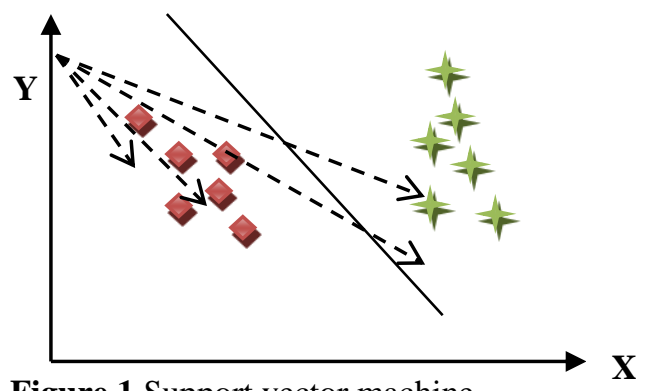

Figure 1 Support vector machine

SVM is treated as a supervised machine learning algorithm (shown in Figure 1). It is used for both classification and regression type problems. In this algorithm, we plot each data item as a point in ndimensional space (where $\mathrm{n}$ is number of features you have) with the value of each feature being the value of a particular coordinate and then classify the same by finding the hyper-plane that differentiate the two classes very well. Support vectors are simply the co-ordinates of individual observation $[15,16]$. Support Vector Machine is a frontier which best segregates the two classes (hyper-plane/ line).The SVM algorithm developed by Vapnik, is based on statistical learning theory. It continues to be one of the most successful algorithms for classification [13, 17]. SVM address the classification problem by finding the hyper plane in the feature space that achieves maximum sample margin when the training samples are separable. Maximum margin can be achieved by minimizing the norm of the classifier [18].Such a hyper plane with maximum margin is likely to be generalized better and are supposed to classify "unseen" or testing data points correctly. Figure 2 shows two groups of data and a separating hyper plane with maximum margin.

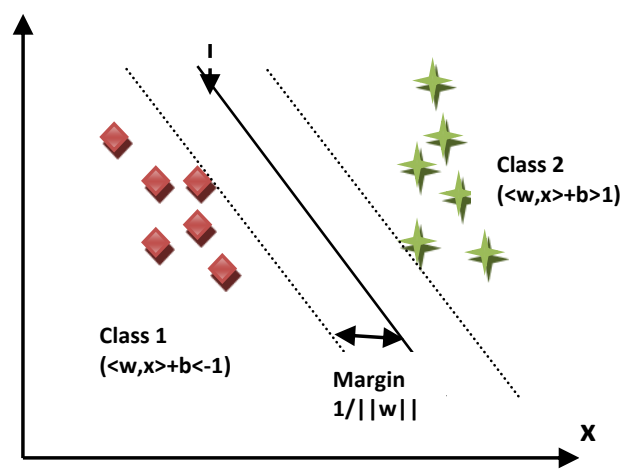

Figure 2 A SVM with a maximum margin classifier

Linear SVMs are better suited for linearly separable data points. They actually try to find a hyperplane $\mathrm{w}^{\mathrm{T}}$ $\mathrm{x}+\mathrm{b}=0$ which separates two classes of training data from each other by satisfying the constraints $\left[\mathrm{w}^{\mathrm{T}} \mathrm{x}_{\mathrm{i}}+\mathrm{b}\right.$ $\geq 1, \forall i$ where $\left.y_{i}=1\right]$ and $\left[w^{T} x_{i}+b \leq-1, \forall i\right.$ where $y_{i}$ $=-1]$. These can be combined into one set of inequalities:

$\mathrm{y}_{\mathrm{i}}\left(\mathrm{w}^{\mathrm{T}} \mathrm{x}_{\mathrm{i}}+\mathrm{b}\right) \geq 1 \forall \mathrm{i}=\{1, \ldots, \mathrm{n}\}$

The distance between two hyperplanes $\left(w^{T} x_{i}+b=1\right.$ and $\left.\mathrm{w}>\mathrm{x}_{\mathrm{i}}+\mathrm{b}=-1\right)$ is $\frac{2}{\|W\|}$ and this is known as margin of the classifier. Hence optimization problem which maximizes the margin along with satisfying the above constraints is

$$
\begin{gathered}
\min \frac{1}{w, b} \| w \\
\text { s.t. } y i\left(\begin{array}{l}
2 \\
\left(w^{T} x_{i}+b\right) \geq 1, i=1, \ldots n
\end{array}\right.
\end{gathered}
$$


The decision function is $\mathrm{f}(\mathrm{x})=\operatorname{sign}\left(\mathrm{w}^{\mathrm{T}} \mathrm{x}+\mathrm{b}\right)$. However, the optimization problem will not have a solution if $\mathrm{D}$ is not linearly separable. To deal with such cases, soft margin SVM has been introduced. It allows mislabeled data points while still maximizing the margin. The method introduces slack variables, $\xi \mathrm{i}$, which measure the degree of misclassification. The following is the optimization problem for soft margin SVM [3].

$$
\begin{gathered}
\min \frac{1}{1}\left\|w \varepsilon_{i} \frac{1}{2}\right\| \frac{2}{2}+c \sum_{i=1}^{n} \varepsilon_{i} \\
\text { s.t. } y i\left(w^{T} x_{i}+b\right) \geq 1-\varepsilon_{i}, \varepsilon_{i} \geq 0, i=1, \ldots \ldots n
\end{gathered}
$$

Here $\mathrm{C}$ is a user-given regularization parameter that determines the trade of between the margin size and the amount of error in training.

\section{Experimental results}

The implementation is done in MATLAB using SVM algorithm, first we selected test database image with the respective number for matching, second we selected train database as the same and the positive samples and negative samples results are shown in Figures 3,4 and 5.

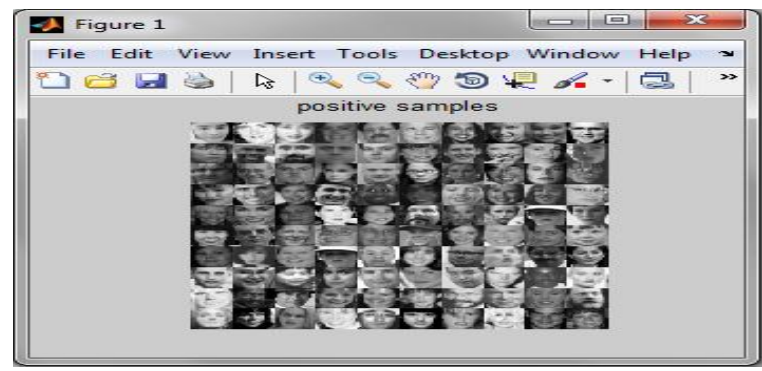

Figure 3 Image of positive samples

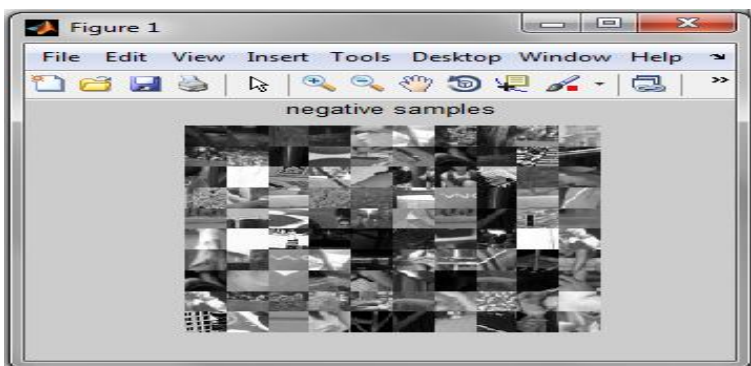

Figure 4 Image of negative samples

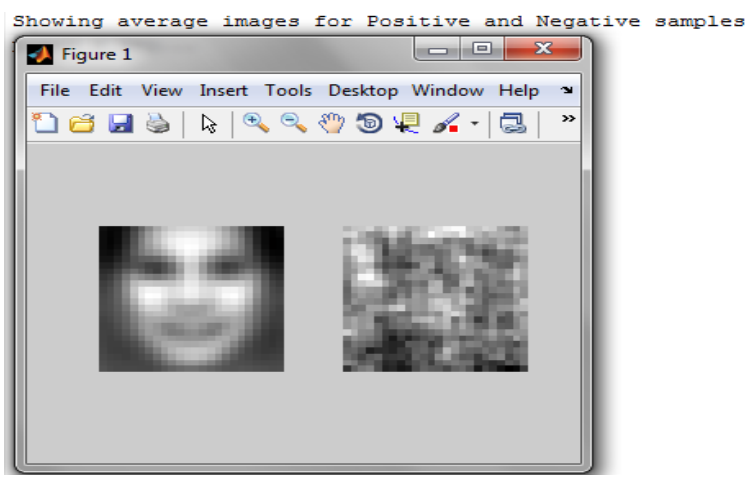

Figure 5 Average images of positive and negative samples

Training SVM classifier with 1000 positive samples and 1000 negative samples, 638 support vectors ,Running evaluation:4.2sec., Training accuracy: 0.940 and validation accuracy: 0.895 .

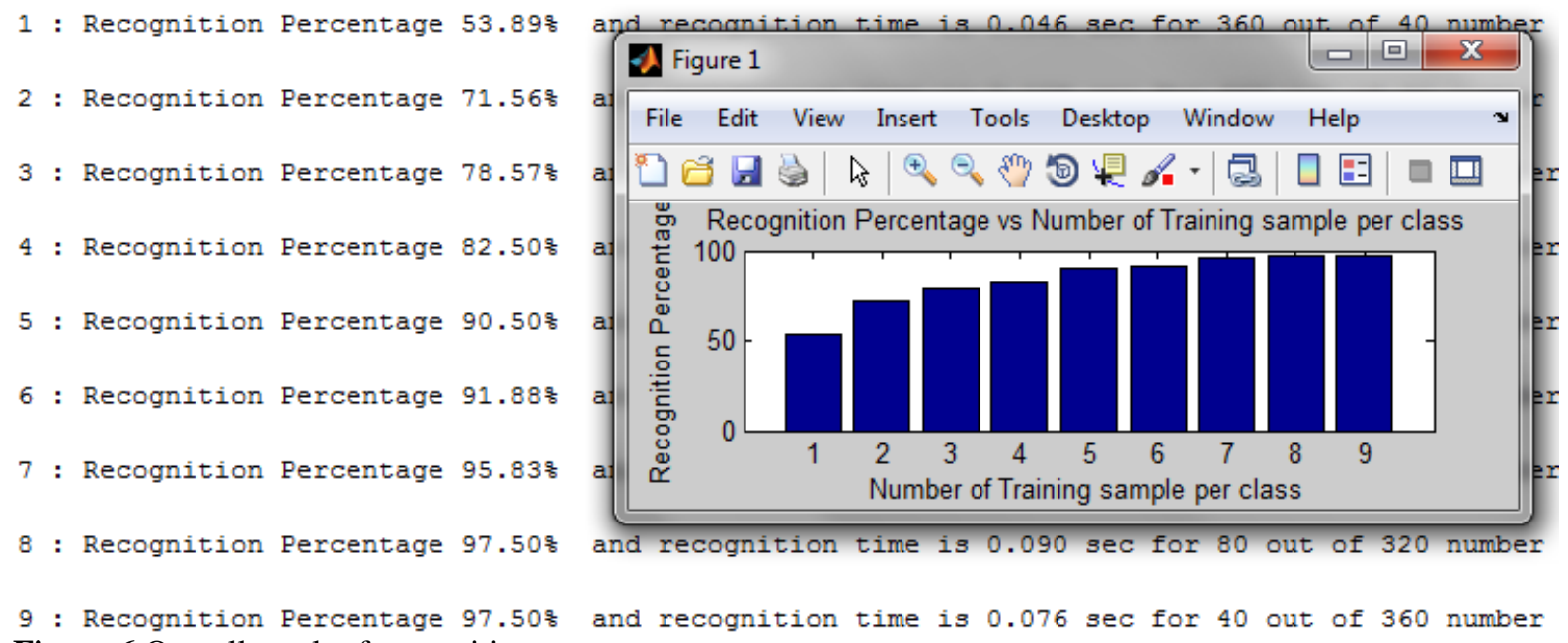

Figure 6 Overall result of recognition percentage 


\section{Conclusion}

In this research paper SVM algorithm is tested for positive and negative samples using training and testing data. The recognition percentage of the algorithm is also shown along with the respective time in Figure 6. The Results of recognition experiments for SVM based faces approach is a wellestablished technique for performing the recognition task, it is more meaningful to compare it with positive and negative samples and finial SVM have good recognition percentage for image.

\section{Acknowledgment}

I like to acknowledge the library of Coimbatore Institute of Technology for providing the facilities for accessing IEEE papers.

\section{Conflicts of interest}

The authors have no conflicts of interest to declare.

\section{References}

[1] Chellappa R, Wilson CL, Sirohey S. Human and machine recognition of faces: a survey. Proceedings of the IEEE.1995; 83(5):705-41.

[2] Zhao W, Chellappa R, Phillips PJ, Rosenfeld A. Face recognition: A literature survey. ACM Computing Surveys. 2003; 35(4):399-458.

[3] Zhi-fang L, Zhi-sheng Y, Jain AK, Yun-qiong W. Face detection and facial feature extraction in color image. In international conference on computational intelligence and multimedia applications 2003(pp. 126-30). IEEE.

[4] Jafri R, Arabnia HR. A survey of face recognition techniques. JIPS. 2009; 5(2):41-68.

[5] Shan C, Gong S, McOwan PW. Facial expression recognition based on local binary patterns: A comprehensive study. Image and Vision Computing. 2009; 27(6):803-16.

[6] Pissarenko D. Eigenface-based facial recognition. Voil. 2002; 1: 4-9.
[7] Shah JD, Patil SH. Biometric authentication based on detection and recognition of multiple faces in image. UFL \& JIITU, IC3-2008 (pp. 87-96). 2008.

[8] Slavković M, Jevtić D. Face recognition using eigenface approach. Serbian Journal of Electrical Engineering. 2012; 9(1):121-30.

[9] Karatzoglou A, Meyer D, Hornik K. Support vector machines in R. 2005.

[10] Kherchaoui S, Houacine A. Face detection based on a model of the skin color with constraints and template matching. In international conference on machine and web intelligence (ICMWI) 2010 (pp. 469-72). IEEE.

[11] Krishnan N, Jothi GR, Aaron GL. Recognition of nonsymmetric faces using principal component analysis. In ICVGIP 2004 (pp. 467-72).

[12] http://www.face-rec.org/journalsbooks/Delac_Grgic_Bartlett_Recent_Advances_in_Fa ce_Recognition.pdf. Accessed 12 May 2016.

[13] Valstar MF, Pantic M. Combined support vector machines and hidden markov models for modeling facial action temporal dynamics. In international workshop on human-computer interaction 2007 (pp. 118-27). Springer Berlin Heidelberg.

[14] Burges CJ. A tutorial on support vector machines for pattern recognition. Data Mining and Knowledge Discovery. 1998; 2(2):121-67.

[15] Zhou P, Ye W, Xia Y, Wang Q. An improved canny algorithm for edge detection. Journal of Computational Information Systems. 2011; 7(5):151623.

[16] Turk MA, Pentland AP. Face recognition using eigenfaces. In IEEE computer society conference on computer vision and pattern recognition 1991 (pp. 586-91). IEEE.

[17] Gupta S, Sahoo OP, Goel A, Gupta R. A new optimized approach to face recognition using eigenfaces. Global Journal of Computer Science and Technology. 2010; 10(1).

[18] Lata YV, Tungathurthi CK, Rao HR, Govardhan A, Reddy LP. Facial recognition using eigenfaces by PCA. International Journal of Recent Trends in Engineering. 2009; 1(1):587-90. 